\title{
Detection and Analysis of Heart Rate Variability in Young and Postmenopausal women in Different Postures
}

\author{
Kirti Rawal", B. S. Saini, and Indu Saini \\ Electronics and Communication Engineering Department, Dr. B R Ambedkar National Institute of Technology, \\ Jalandhar-144011, Punjab, India \\ * e-mail: nitkr1234@gmail.com
}

\begin{abstract}
Postmenopause is the naturally occurring stage in woman's life after the permanent cessation of the menstruation longer than 12 months. The transition from the young to the postmenopausal stage impacts the variation in the heart rate. It is important to analyze and detect the variation in Heart Rate Variability (HRV) between young and postmenopausal women in different postures to understand the impact of the age and physical activities on the autonomic health of heart. The aim of this study is to (i) investigate the effect of autonomic regulations of heart rate in young and postmenopausal women in various postures and (ii) determine the possibilities for the reliable, efficient and accurate HRV analysis signal processing algorithm in detecting HRV variations between young and postmenopausal women. The experiments were performed on 25 young women and 25 old women in the postmenopausal phase. Various linear methods have been applied for analysing and detecting HRV variations between young and postmenopausal women. The results concluded that the postmenopausal group showed significantly lower $(\mathrm{p}<0.05)$ values of SDNN, NN50, pNN50, RMSSD, and HRVTi than young group both in the lying and standing postures. These results indicate a decrease in the parasympathetic activity in postmenopausal as compared to the young women due to the age. Further, the performance of linear methods in detecting HRV variations between postmenopausal and young women in different postures was calculated. It is also concluded from the results that HRVi outperforms other HRV analysis methods in detecting HRV variations between postmenopausal and young women. (C) 2016 Journal of Biomedical Photonics \& Engineering.
\end{abstract}

Keywords: Follicular phase, heart rate variability, linear methods, menstrual cycle, postmenopause, postures.

Paper \#2767 received 2015.11.23; revised manuscript received 2016.01.09; accepted for publication 2016.01.09; published online 2016.03.04. doi: 10.18287/JBPE16.02.020301

\section{References}

1. D. T. Pace, "Effect of Postmenopausal Hormone Replacement Therapy on Heart Rate Variability," Ph. D. Thesis, University of Tennessee, Memphis (1998).

2. T. R. Johnson, "Knowledge and Attitudes Regarding the Menstrual Cycle, Oral Contraceptives, and Sport Performance: The Conceptualization and Development of a Questionnaire for Athletic Coaches," Ph. D. Thesis, Florida State University, Florida (2008).

3. S. Moodithaya, and T. Sandhya Avadhany, "Comparison of Cardiac Autonomic Activity Between Pre And Post Menopausal Women Using Heart Rate Variability,” Indian J Physiol Pharmacol 53, 227-234 (2009).

4. H. C. Souza, and G. C. Tezini, "Autonomic cardiovascular damage during post menopause: the role of Physical Training," Aging Dis 4(6), 320-328 (2013). 
5. V. F. Neves, M. F. Silva de Sa, L. Jr. Gallo, and A. M. Catai, et al., "Autonomic modulation of heart rate of young and postmenopausal women undergoing estrogen therapy," Braz J Med Biol Res 40(4), 491-499 (2007).

6. N. H. Farag, R. A. Nelesen, B. L. Parry, J. S. Loredo, et al., "Autonomic and cardiovascular function in postmenopausal women: the effects of estrogen versus combination therapy," Am J Obstet Gynecol 186(5), 954-961 (2002).

7. G. M. Rosano, R. Patrizi, F. Leonardo, P. Ponikowski, et al., "Effect of estrogen replacement therapy on heart rate variability and heart rate in healthy postmenopausal women," Am J Cardiol 80(6), 815-817 (1997).

8. O. Rosa Brito-Zurita, C. Posadas-Romero, A. G. Hermosillo, J. Zamora-Gonzalez, et al., "Estrogen effect on heart rate variability in hypertensive postmenopausal women," Maturitas 44(1), 39-48 (2003).

9. P. Tuomikoski, O. Ylikorkala, and T. S. Mikkola, "Menopausal hot flushes and vascular health," Ann Med 43(4), 283-291 (2011).

10. T. Beljic, D. Babic, J. Marinkovic, and G. M. Prelevic, "Effect of estrogen replacement therapy on cardiac function in postmenopausal women with and without flushes," Gynecol Endocrinol 13(2), 104-112 (1999).

11. H. Hautamaki, P. Haapalahti, P. Piirila, P. Tuomikoski, et al., "Effect of hot flushes on cardiovascular autonomic responsiveness: A randomized controlled trial on hormone therapy," Maturitas 72(3), 243-48 (2012).

12. J. O. Lee, S. G. Kang, S. H. Kim, S. J. Park, et al., "The Relationship between Menopausal Symptoms and Heart Rate Variability in Middle Aged Women," Korean J Fam Med 32(5), 299-305 (2011).

13. C. L. Brockbank, F. Chatterjee, S. A. Bruce, and R. C. Woledge, "Heart rate and its variability change after the menopause," Exp Physiol 85(3), 327-330 (2000).

14. Task force of the European society of cardiology and the North American society of pacing and electrophysiology, "Heart rate variability - standards of measurement. Physiological interpretation and clinical use," Circulation 93(5), 1043 - 1065 (1996).

\section{Introduction}

Every women experience the menstrual cycle and menopause in its life. The menstrual phase, luteal phase and follicular phase are the three phases of the menstrual cycle. Out of these phases, follicular phase is the phase which starts after the end of menstrual phase where the hormonal variations are not affected by progesterone. The menstrual cycle ceases at an approximate age of 45 to 50 years which is commonly termed as menopause. The termination of menstruation continuously longer than 12 months is postmenopause. During the menstrual cycle and postmenopause, there are many physiological changes occur in women that affect the cardiac functioning of the heart [1-2]. As the women age increases from young towards the menopause and to the postmenopause, the risk towards the cardiovascular diseases also increases. Moreover, every women experience an extensive fluctuation in HRV during the menopause and even after the menopause. Thus, it is important to study the impact of age and posture on the cardiac functioning of the heart.

Moodithaya et al. [3] compared the HRV variations in premenopausal and postmenopausal women. Souza et al. [4] reviewed the influence of cardiovascular diseases and its association with physical training in postmenopausal state of women. Neves et al. [5] compared the autonomic regulations of heart rate in young and postmenopausal women. Farag et al. [6], Rosano et al. [7] and Rosa Brito-Zurita et al. [8] investigated the effect of estrogen therapy on the autonomic modulation of heart rate. Tuomikoski et al. [9], Beljic et al. [10], Huatamaki et al. [11] and Lee et al. [12] evaluated the effect of hot flushes in the cardiac functioning of postmenopausal women. Brockbank et al. [13] reported decreased linear HRV parameters in the postmenopausal women rather than premenopausal women.

The variation in HRV is linked with the development of heart diseases. The cardiovascular diseases are considered to be the prime reason of mortality in the postmenopausal women. Therefore, it is important to analyze and detect the variations in HRV between the postmenopausal and young women in order to have the insight of heart, so that the proper counseling and prescription can be given to the women.

After an extensive literature survey, it has been observed that there are certain shortcomings in the existing studies in the analysis and detection of HRV in young and postmenopausal women [14]. Following are the shortcomings of the existing studies:

1. To the best of author's, there is no substantial work that delves down to the details of HRV analysis in young and postmenopausal phase of women in the lying and standing postures.

2. There is no such study that exhibits the performance of HRV analysis methods in detecting HRV variations between the young and postmenopausal women in the lying and standing postures.

So, decoding HRV variations using linear methods of HRV during the postmenopause and young women proves to be crucial in determining the health conditions of women and also necessary to decide course of action for treatment of various types of diseases. In other words, the analysis and detection of HRV variations between the postmenopausal and young women can be used to evaluate the adaptations of autonomic 
regulations of heart in the young and old women in different postures.

The objective of the present study is to compare and detect heart rate variations between the postmenopausal and young women in the lying and standing postures.

In linear methods, first the time domain methods such as Standard Deviation of NN interval (SDNN), number of pairs of adjacent $\mathrm{NN}$ intervals differing by more than 50ms (NN50), percentage of number of pairs of adjacent Normal to Normal intervals differing by more than $50 \mathrm{~ms}$ (pNN50), Root Mean Square of Successive Differences (RMSSD), mean Heart Rate (HR), HRV Triangular Index (HRVTi) have been implemented on preprocessed HRV dataset of 25 old women in the postmenopause and 25 young women in the lying and standing postures separately. Similarly, frequency domain methods such as Low Frequency/ High Frequency (LF/HF ratio) have been applied on same data set. The results indicate that there is decrease in the parasympathetic activity in postmenopausal women as compared to the young women in the lying and standing postures. It is also concluded from the results that HRVTi performs better than other HRV analysis methods in detecting HRV variations between the postmenopausal and young women.

\section{Data Description}

In this paper, two ECG data sets were used to evaluate the HRV variations between the young and postmenopausal women. First dataset consist of 25 young healthy women in the follicular phase of the menstrual cycle having age group of 18-24 years has been recorded using BIOPAC and second dataset of 25 old healthy women with postmenopause phase having age group of 45-55 years in the lying and standing postures.

ECG dataset has been acquired using BIOPAC ${ }^{\circledR}$ MP150 system at sampling frequency of $500 \mathrm{~Hz}$. Software Acknowledge 4.2 has been used for acquisition of HRV signals of all subjects. All ECG recordings were performed for 15 minutes duration. After each recording of 15 minutes in lying posture, subject rested for 10 minutes before beginning another 15 minutes recording in standing posture. The written consent was taken from all subjects.

\section{Results and Discussion}

The present research work is expected to (i) find the autonomic regulations of heart in the young and postmenopausal women in different postures and (ii) to evaluate HRV analysis methods that can detect the HRV variations between the young women and postmenopausal women in different postures.

In order to analyze the HRV between the young and postmenopausal women, the results are presented in box plots as median, $1^{\text {st }}$ and $3^{\text {rd }}$ quartiles and outliers. The postmenopausal and young women data set was statistically analyzed using non parametric Mann-
Whitney test comparison. The level of significance was set at $\mathrm{p}<0.05$.

Secondly, the performance evaluation of HRV analysis methods in detecting HRV variations between the postmenopausal and young women is done using accuracy as statistical parameter.

Fig. 1 to Fig. 7 illustrates that analysis of HRV expressed as various time and frequency domain indexes (SDNN, NN50, pNN50, RMSSD, HRVTi, mean $\mathrm{HR}$ and $\mathrm{LF} / \mathrm{HF}$ ratio) obtained for postmenopausal and young women data sets in the lying and standing postures. It is shown in Fig. 1 to Fig. 5 that there is significant difference $(\mathrm{P}<0.05)$, with lower values of indexes like SDNN, NN50, pNN50, RMSSD, HRVTi in the postmenopausal women as compared to the young women in the lying as well as in standing postures. Further, Fig. 6 and Fig. 7 shows that there is no significant difference $(\mathrm{P}>0.05)$ in Mean $\mathrm{HR}$ and $\mathrm{LF} / \mathrm{HF}$ ratio in the lying and standing postures.

The comparative analysis of various time and frequency domain HRV indexes of postmenopausal and young women in the lying and standing postures is shown in Table 1.

The results demonstrated in Table 1 states that mean and Standard Deviation (SD) of all the parameters of linear methods like SDNN, NN50, pNN50, RMSSD, and HRVTi in postmenopausal in the lying as well in standing is less than corresponding mean values of the young women. On the other hand, the mean LFHF ratio in young women comes out to be less than the corresponding mean LFHF ratio of postmenopausal phase both in the lying and standing postures. But, mean HR of postmenopausal women is higher in the lying and lower in the standing posture as compared to the young women.

Thus, based on the results shown in Fig. 1 to 7 and Table 1 , it is observed that there is significant decrease in HRV in the postmenopausal women in the lying and standing postures as compared to the young women, i.e., $H R V_{P}<H R V_{Y}$ where $H R V_{P}$ is the value of HRV in the postmenopausal stage of women and $H R V_{Y}$ is the value of HRV in the young women.

The prime reason can be the influence of age and relatively low physical activities in old women.

Secondly, various linear HRV analysis methods (Time domain and frequency domain) are used to detect the HRV variations between the postmenopausal and young women in the lying and standing postures. This is done using statistical performance evaluator. This statistical parameter is known as accuracy and is defined using following:-

The accuracy is defined as the ability of HRV method to detect correct HRV variations between the postmenopausal and young women. Each group (postmenopausal $\left(N_{P}\right)$ and young women $\left(N_{Y}\right)$ ) consists of 25 subjects i.e. $N_{P}=25$ and $N_{Y}=25$ respectively. For a specific HRV method, each value of postmenopausal is compared with each value of young women. Then, based on the correct relationship of HRVs between the postmenopausal and young women true cases are 


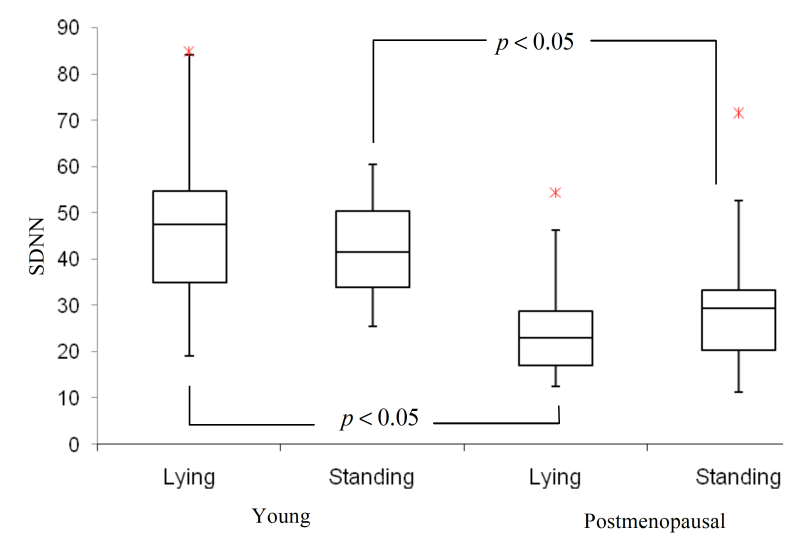

Fig. 1 Comaprative analysis of young and postmenopausal women using SDNN.

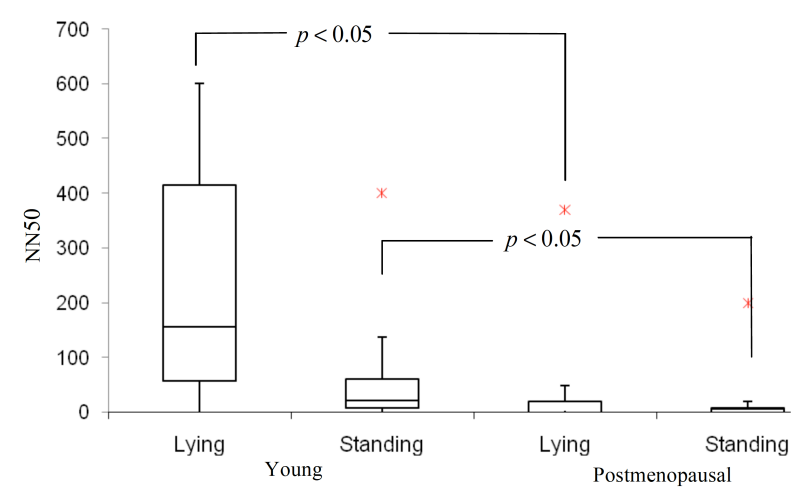

Fig. 2 Comaprative analysis of young and postmenopausal women using NN50.

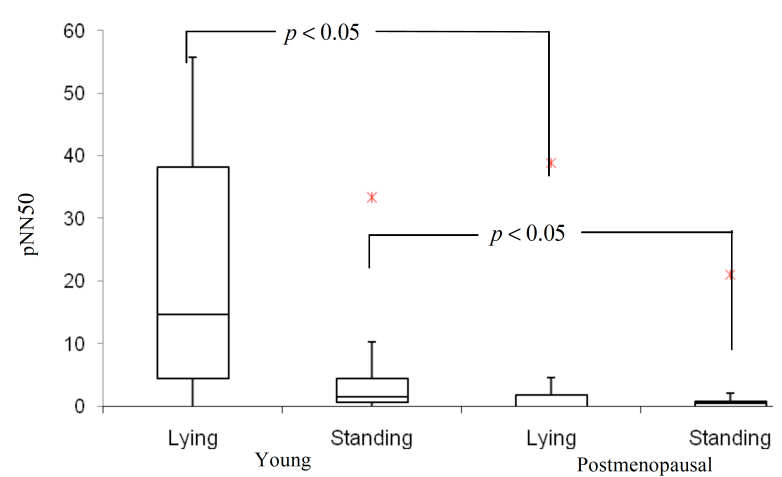

Fig. 3 Comaprative analysis of young and postmenopausal women using pNN50.

calculated by summing all true cases. Then, by dividing the true cases by total number of comparisons $\left(N=N_{P} * N_{Y}\right)$.

Thus based on the above mentioned explanation, the accuracy is defined as follows:

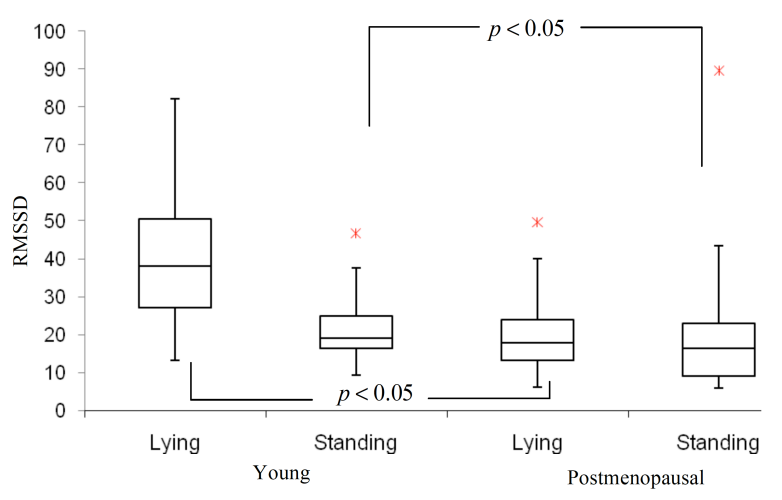

Fig. 4 Comaprative analysis of young and postmenopausal women using RMSSD.

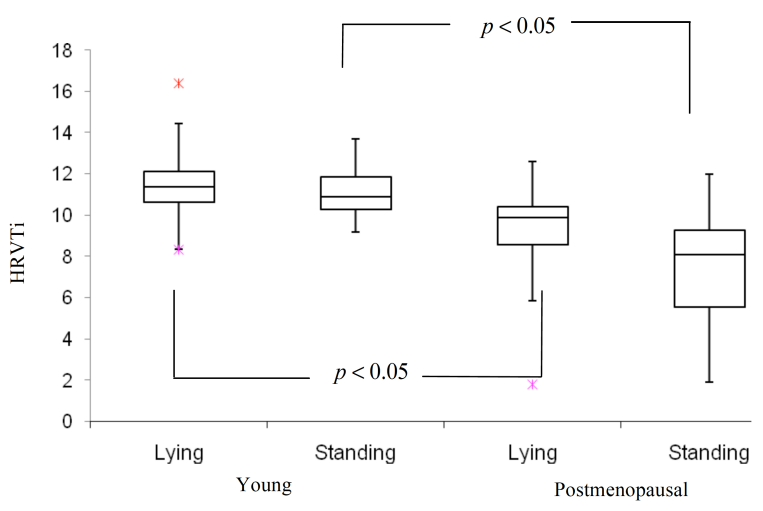

Fig. 5 Comaprative analysis of young and postmenopausal women using HRVTi.

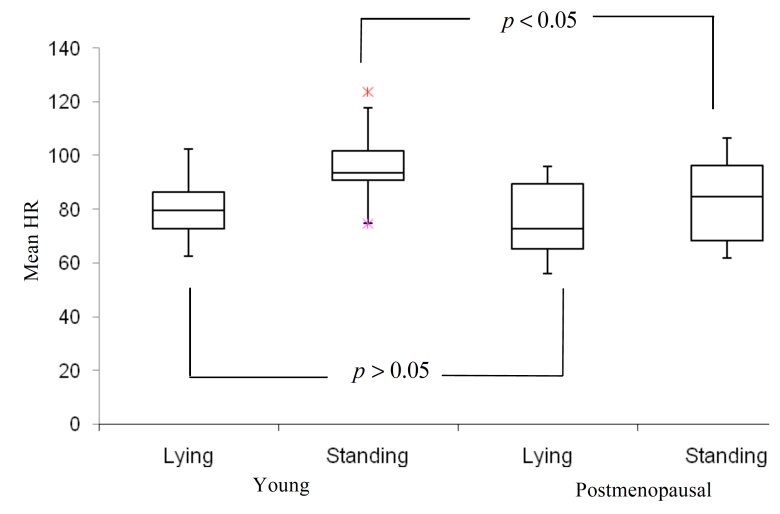

Fig. 6 Comaprative analysis of young and postmenopausal women using mean HR.

$$
\text { Accuracy }=\frac{\sum_{h=1}^{N} T S_{h}}{N},
$$

where $T S_{h}=1$ (Truecases), if correct relationship between postmenopausal and young women is detected; 
Table 1 Mean and standard deviation values of Linear Methods of HRV in young and postmenopausal women in lying and standing postures.

\begin{tabular}{cccccc} 
& HRV Analysis & \multicolumn{2}{c}{ Lying } & \multicolumn{2}{c}{ Standing } \\
\cline { 3 - 6 } S. No & Methods & Young Women & Postmenopausal women & Young Women & Postmenopausal women \\
\hline 1. & SDNN & $47.266 \pm 15.432$ & $24.075 \pm 11.373$ & $43.987 \pm 11.199$ & $30.170 \pm 16.324$ \\
\hline 2. & NN50 & $264.208 \pm 212.079$ & $28.958 \pm 78.939$ & $74.333 \pm 114.78$ & $22.291 \pm 45.612$ \\
\hline 3. & pNN50 & $23.562 \pm 19.237$ & $3 \pm 8.259$ & $5.962 \pm 9.693$ & $2.983 \pm 6.067$ \\
\hline 4. & RMSSD & $42.512 \pm 18.397$ & $18.137 \pm 9.971$ & $23.241 \pm 10.680$ & $22.420 \pm 22.480$ \\
\hline 5. & meanHR & $78.229 \pm 10.425$ & $78.429 \pm 12.924$ & $93.820 \pm 10.378$ & $84.016 \pm 15.198$ \\
\hline 6. & HRVTi & $11.479 \pm 1.676$ & $9.433 \pm 2.045$ & $11.095 \pm 1.201$ & $7.212 \pm 2.737$ \\
\hline 7. & LF/HF ratio & $1.243 \pm 1.646$ & $2.341 \pm 2.646$ & $2.757 \pm 1.360$ & $5.453 \pm 7.982$
\end{tabular}

$T S_{h}=0($ otherwise $) ; \sum_{h=1}^{N} T S_{h}$ is the total number of true cases.

The HRV method is considered as accurate if for each subject the correct relationship of HRV variation between the postmenopausal and young women, i.e., $H R V_{P}<H R V_{Y}$ (This relationship is established using Fig.1 to Fig.7) is detected. The accuracy of various linear methods in detecting HRV variations between postmenopausal and young women in the lying posture is shown in Fig. 8.

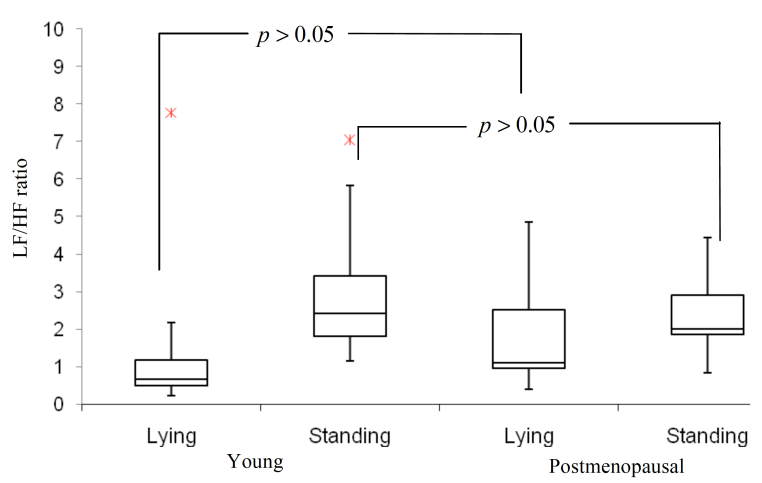

Fig. 7 Comaprative analysis of young and postmenopausal women using LF/HF ratio.

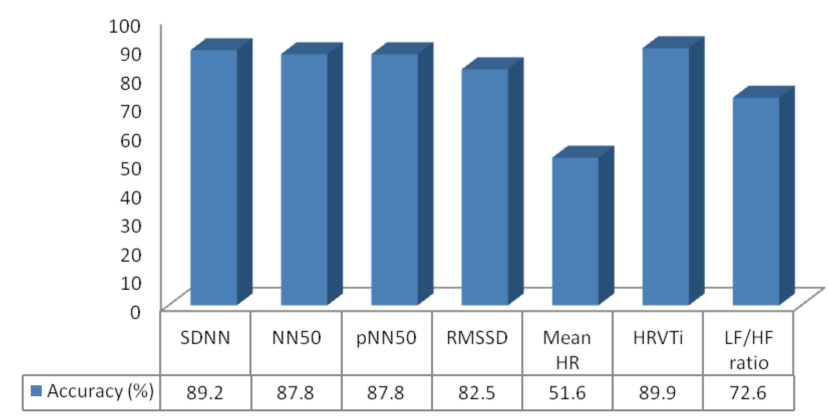

Fig. 8 Comparative analysis of linear methods of HRV in young and postmenopausal women in the lying posture.

From Fig. 8 and Fig.9, it is amply clear that HRVTi is having higher accuracy in detecting HRV variations between the postmenopausal and young women in the lying and standing postures. Thus, it is concluded from Fig. 8 and Fig. 9 that HRVTi performs better than other linear methods in detecting HRV variations between young and postmenopausal women in the lying as well in standing postures.

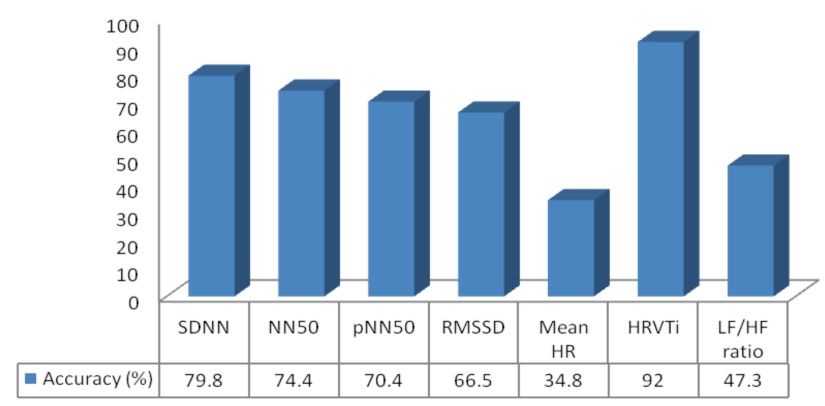

Fig. 9 Comparative analysis of linear methods of HRV in young and postmenopausal women in the standing posture.

\section{Conclusion}

HRV is a non-invasive approach that is used to provide information about the autonomic regulation of heart in young and postmenopausal women. The menopausal transitions are commonly associated with fluctuations in cardiac activity. This study was expected to (i) compare the variations of heart rate in postmenopausal and young women in the lying and standing postures and (ii) assess the performance of overall accuracy of each linear method for detecting HRV variations between the young women and postmenopausal women. It is concluded from the results that there is reduction in HRV in the postmenopausal women as compared to the young women both in the lying and standing postures. This reduction may be due to the age and less agility in old age. It is also concluded that HRVTi is able to detect HRV variations between the young and postmenopausal women better than other linear HRV analysis methods. The study can be extended to apply nonlinear methods for HRV analysis and to improve the accuracy of detecting HRV variations between postmenopausal and young women. 